\title{
Characterization of whole genome amplified (WGA) DNA for use in genotyping assay development
}

Tao Han ${ }^{1 *}$, Ching-Wei Chang ${ }^{2}$, Joshua C Kwekel', Ying Chen ${ }^{3}$, Yun Ge ${ }^{1}$, Francisco Martinez-Murillo ${ }^{4}$, Donna Roscoe ${ }^{4}$, Živana Težak ${ }^{4}$, Reena Philip ${ }^{4}$, Karen Bijwaard ${ }^{4}$ and James C Fuscoe ${ }^{1 *}$

\begin{abstract}
Background: Genotyping assays often require substantial amounts of DNA. To overcome the problem of limiting amounts of available DNA, Whole Genome Amplification (WGA) methods have been developed. The multiple displacement amplification (MDA) method using $\$ 29$ polymerase has become the preferred choice due to its high processivity and low error rate. However, the uniformity and fidelity of the amplification process across the genome has not been extensively characterized.

Results: To assess amplification uniformity, we used array-based comparative genomic hybridization (aCGH) to evaluate DNA copy number variations (CNVs) in DNAs amplified by two MDA kits: GenomiPhi and REPLI-g. The Agilent Human CGH array containing nearly one million probes was used in this study together with DNAs from a normal subject and 2 cystic fibrosis (CF) patients. Each DNA sample was amplified 4 independent times and compared to its native unamplified DNA. Komogorov distances and Phi correlations showed a high consistency within each sample group. Less than $2 \%$ of the probes showed more than 2-fold CNV introduced by the amplification process. The two amplification kits, REPLI-g and GenomiPhi, generate very similar amplified DNA samples despite the differences between the unamplified and amplified DNA samples. The results from aCGH analysis indicated that there were no obvious CNVs in the CFTR gene region due to WGA when compared to unamplified DNA. This was confirmed by quantitative real-time PCR copy number assays at 10 locations within the CFTR gene. DNA sequencing analysis of a 2-kb region within the CFTR gene showed no mutations introduced by WGA.
\end{abstract}

Conclusion: The relatively high uniformity and consistency of the WGA process, coupled with the low replication error rate, suggests that WGA DNA may be suitable for accurate genotyping. Regions of the genome that were consistently under-amplified were found to contain higher than average GC content. Because of the consistent differences between the WGA DNA and the native unamplified DNA, characterization of the genomic region of interest, as described here, will be necessary to ensure the reliability of genotyping results from WGA DNA.

Keywords: Whole genome amplification, Array-based comparative genomic hybridization, TaqMan copy number assay, DNA sequencing

\footnotetext{
* Correspondence: Tao.Han@fda.hhs.gov; James.Fuscoe@fda.hhs.gov

'Division of Systems Biology, National Center for Toxicological Research, FDA, Jefferson, AR 72079, USA

Full list of author information is available at the end of the article
} 


\section{Background}

Advances in genomic technologies have enabled development of many novel genome analysis methods that may have applications in the understanding, diagnosis, and management of genetic diseases and cancer. Comprehensive high-throughput assays are available for detection of single nucleotide polymorphisms (SNPs) [1-3], DNA copy number variation (CNV) [4], microsatellite expansion or contraction [5] and loss of heterozygosity (LOH) [6], all of which detect sometimes subtle genomic alterations associated with disease. Some of these assays require micrograms of DNA which may be difficult to obtain for many clinical samples. In addition, inadequate DNA template may prevent the performance of multiple assays on a single sample [7]. Limited availability of DNA also poses challenges for manufacturers and regulators of genetic diagnostic devices. One of these challenges is availability of sufficient quantities of DNA samples to appropriately validate a particular test under investigation, especially when uncommon mutations for a rare disease severely limit access to patient sample specimens.

Since the U.S. Food and Drug Administration (FDA)'s clearance of the first genotyping assay for cystic fibrosis (CF) in 2005, the Office of In Vitro Diagnostic Device Evaluation and Safety within the Center for Devices and Radiological Health (CDRH) has reviewed a number of other genotyping assays for inherited disorders. Some of these genotyping assays are intended to detect relatively rare heritable diseases consisting of multiple disease-causing alleles (mutations) for each disease, while others may detect more common diseases, but are intended to test very rare as well as common mutations. To show the accuracy of these assays, manufacturers would generally use patient samples (whole blood or archived DNA). For rare mutations or alleles, it is often difficult to obtain sufficient quantities of such clinical samples to adequately assess test performance. Therefore, there is an interest in using whole genome amplified (WGA) DNA samples created from patient samples instead of native DNA samples to increase the availability of appropriate samples to query the performance of the assays. WGA is a method that amplifies small amounts of genomic DNA several thousand-fold in vitro. The WGA process has the potential, however, to result in non-uniform amplification of the DNA in which some regions of the genome are overrepresented and others are under-represented. Such biased amplification could make the WGA DNA unsuitable for some of the studies designed to assess the clinical assay performance.

Several WGA methods based on the polymerase chain reaction (PCR) with Taq polymerase were initially developed [8-12]. These methods included the use of primers directed at highly repetitive sequences [11], ligation of linkers to fragmented DNA [12], degenerate oligonucleotide primed PCR [9], and primer extension preamplification [10]. All of these methods suffer from a relatively high level of mutations in the amplified DNA (error rate $3 \times 10^{-5}[13]$ ) and highly non-uniform amplification due to the low fidelity and low processivity of the Taq polymerase, respectively. In 2002, Dean et al. [14] described the multiple displacement amplification (MDA) technique. This method of WGA takes advantage of the high processivity and low error rate of the Ф29 bacteriophage DNA polymerase. This polymerase has a 3'-5' proof-reading activity and adds an average of 70,000 templated nucleotides to a primer [15], resulting in higher fidelity and less biased amplification than with the Taq polymerase methods. The WGA process using Ф29 polymerase is isothermal and uses random primers to target the entire genome. The polymerase has strong strand displacement activity so that exponential amplification occurs through a branching mechanism [14], resulting in a high yield of DNA. The MDA process has been recently reviewed and shown to be superior to other DNA amplification methods with regard to genotyping, genomic coverage, and amplification bias [16,17].

MDA-based WGA has been frequently used in DNA sample preparation for genotyping and sequencing in recent years [18-21]. High call rates (97.5\%) and excellent concordance rates were achieved from WGA samples using high-density SNP arrays $[19,20]$. There was a very low error rate (1 SNP genotyping error per 1000 assays) when high quality DNA was used as template [21]. MDA-based WGA has also been used for analyses of single cells [22,23]. Jiang et al. successfully amplified DNA from single sperm at least 250 fold with a single round of MDA [24]. DNA amplified from single lymphocytes was used for multiple analyses of 20 different loci including the $\Delta \mathrm{F} 508$ deletion in exon 10 and two intragenic microsatellite markers in the CF gene [25]. Short tandem repeats (STR) and Human Leucocyte Antigen typing were performed using DNA amplified by MDA from a single cell [26]. MDA-WGA has also been increasingly used in the field of forensic testing $[27,28]$. Even in the often degraded DNA samples, WGA showed the capability and potential to increase the quality and quantity of DNA from difficult samples in forensic casework [29]. Although MDA-based WGA has been successfully applied in many studies, there are several potential problems that may affect interpretation of results. These include the finding that large amounts of nonspecific DNA amplification can be generated during MDA, mostly due to primer-directed DNA synthesis [5,30], and the quantity [21,31] and quality [32] of input DNA into the MDA reaction can affect genotyping results. 
Table 1 DNA sample information

\begin{tabular}{|c|c|c|c|c|c|c|c|}
\hline \multirow[t]{2}{*}{ Sample } & Visit/Blood & \multirow[t]{2}{*}{ Ethnicity } & \multirow[t]{2}{*}{ Sex } & \multirow[t]{2}{*}{ Age } & \multirow[t]{2}{*}{ Genotype } & \multirow[t]{2}{*}{ Diagnosis } & \multirow[t]{2}{*}{$\mathrm{OD}_{260 / 280}$} \\
\hline & $\overline{\text { Draw date }}$ & & & & & & \\
\hline Normal & $9 / 27 / 2002$ & Caucasian & $M$ & 52 & Control & & 1.76 \\
\hline CF1 & 10/30/2003 & Caucasian & M & 26 & $2 \times F 508$ & Cystic Fibrosis & 1.84 \\
\hline \multirow[t]{2}{*}{ CF2 } & 10/30/2003 & Caucasian & $\mathrm{F}$ & 20 & $1 \times G 542 X$ & Cystic Fibrosis & 1.83 \\
\hline & & & & & 1×3659delC & & \\
\hline
\end{tabular}

DNA samples were purchased from PrecisionMed, Inc. and dissolved in TE buffer (10 mM Tris, $1 \mathrm{mM}$ EDTA at pH 8.0). There are different mutations in the two patients with cystic fibrosis (CF1 and CF2).

Despite the advantage of the MDA-based WGA method, the uniformity of the amplification process across the genome has not been extensively characterized [3,4,33]. In 2004, Paez et al. [33] examined DNAs amplified using a commercially available MDA method, REPLI-g, using $10 \mathrm{k}$ Affymetrix SNP arrays and direct sequencing of $\sim 500,000$ bp of DNA and showed nearcomplete genome representation, as well as low replication error rate. In 2006, Pinard et al. [34] assessed the bias of WGA methods on bacterial genomes using massively parallel sequencing and found statistically significant amplification bias, although the MDA methods produced the least bias. Arriola et al. (2007) [35], using a second commercially available MDA method, GenomiPhi, evaluated the WGA DNA with low density array comparative genomic hybridization $(\mathrm{aCGH})$, a method with higher resolution $(\sim 200 \mathrm{~kb}$ in this case $)$ than the traditional CGH method [36]. Copy number biases were found, with the extent dependent on the degree of amplification. While these studies and others [16,17] have consistently shown non-uniformity of amplification by the MDA methods (although less than with other WGA methods), the uniformity of amplification has not been examined with high resolution aCGH at a resolution of several $\mathrm{kb}$. In addition, the consistency of the amplification process has not been evaluated in replicate amplifications of the same sample, an important parameter when such material may be used for validation of genetic diagnostic devices or tests.

In this study, we evaluated two commonly used methods of WGA for their ability to produce large quantities of uniformly amplified DNA with minimal introduced mutations. Biases during the amplification process were determined by measurements of DNA CNVs at nearly 1 million positions in the genome using aCGH analysis. Particular attention was given to a single gene, CFTR, which encodes cystic fibrosis transmembrane conductance regulator. Mutations in CFTR can cause cystic fibrosis, a rare disease manifested by thick, sticky mucus and salty sweat, which usually leads to lung transplant or early death [37]. In addition to $\mathrm{aCGH}$, copy number variation in the CFTR gene was examined by a second method, quantitative real-time PCR. Finally, the introduction of mutations during the WGA process was examined by comparing the DNA sequence of the resulting amplified DNA to that of the unamplified DNA. The consistency of the WGA process was evaluated by examining four replicate amplified DNAs derived from three individuals, one healthy and two with cystic fibrosis.

\section{Results}

\section{CNV introduced by the WGA process}

To characterize the consistency and faithfulness of whole genome amplification methods based on $\Phi 29$

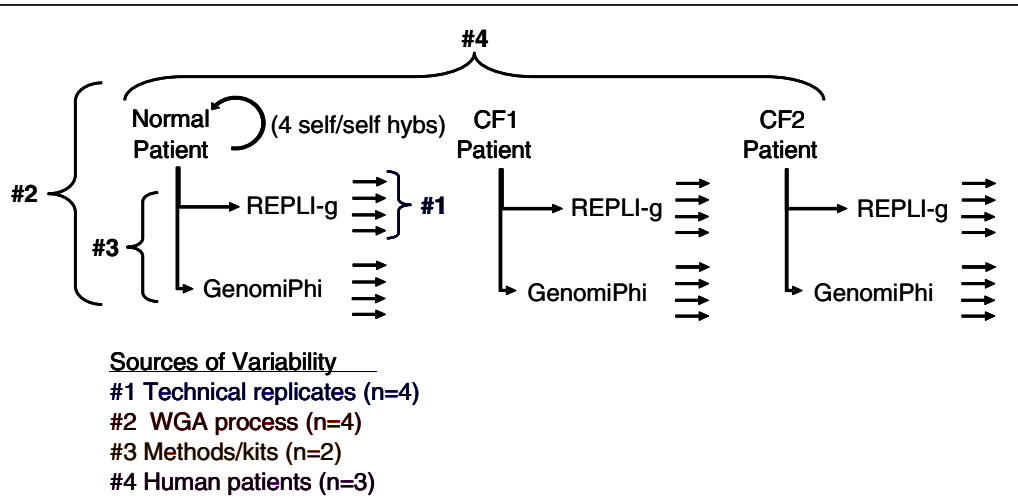

Figure 1 aCGH experimental design with 4 replications each for GenomiPhi and REPLI-g. DNAs used in this study were from one healthy individual (Normal) and two patients with cystic fibrosis (CF1 and CF2). 


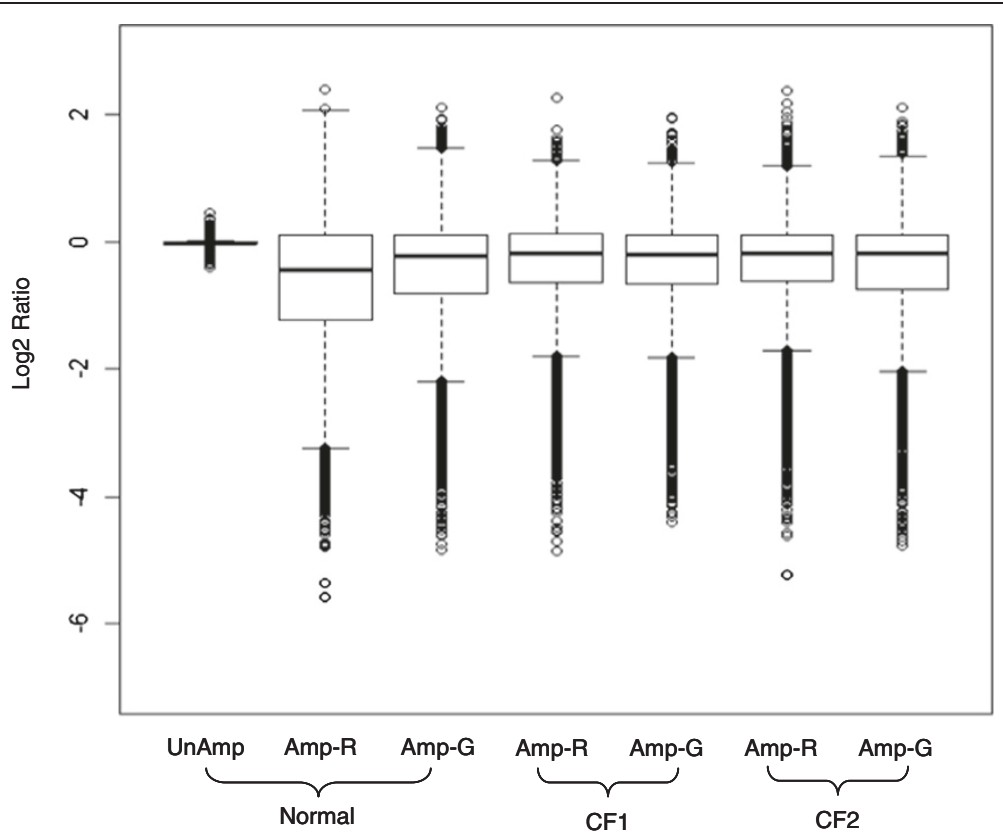

Figure 2 Box plot of log2 ratios of samples to unamplified samples for Normal, CF1, and CF2 samples. The average of the 4 replicates in each sample group is shown. UnAmp (no amplification); Amp-R (REPLI-g amplified); Amp-G (GenomiPhi amplified).

polymerase, three human DNA samples (Table 1) were amplified using two commercially available kits, REPLI-g and GenomiPhi. The DNA samples were derived from a healthy individual (Normal) and two patients with cystic fibrosis (CF1 and CF2). The experimental design is shown in Figure 1. Each of the three DNA samples was amplified 4 independent times by both REPLI-g and GenomiPhi kits. DNA copy number changes introduced into the amplified DNA were evaluated by comparing the amplified DNA to the native unamplified DNA utilizing aCGH. The Agilent Human $\mathrm{CGH}$ array was used with the assumption that as a research tool it could comprehensively evaluate and reveal gross copy number changes at nearly 1 million loci. In addition, unamplified Normal samples were compared to unamplified Normal samples four times to define the technical variability of the aCGH process. Any copy number variation in such a self-self hybridization would be due to technical limits of the aCGH process since the DNA samples were identical. A total of $28 \mathrm{CGH}$ arrays were used in this study.

Figure 2 shows box plots of the average $\log _{2}$ ratios of amplified or unamplified test samples versus the

Table 2 Pair-wise comparison of Kolmogorov distances between probe ratio distributions of unamplified and amplified Normal patient DNA samples

\begin{tabular}{|c|c|c|c|c|c|}
\hline \multicolumn{2}{|c|}{ Sample } & \multicolumn{4}{|c|}{ Kolmogorov distance } \\
\hline & & \multicolumn{4}{|c|}{ Normal UnAmp/UnAmp } \\
\hline & & Replicate 1 & Replicate 2 & Replicate 3 & Replicate 4 \\
\hline \multirow[t]{4}{*}{ NormalAmp-R/ UnAmp } & Replicate 1 & 0.398 & 0.402 & 0.405 & 0.402 \\
\hline & Replicate 2 & 0.409 & 0.413 & 0.416 & 0.413 \\
\hline & Replicate 3 & 0.385 & 0.389 & 0.392 & 0.388 \\
\hline & Replicate 4 & 0.415 & 0.419 & 0.423 & 0.420 \\
\hline \multirow[t]{4}{*}{ Normal Amp-G/UnAmp } & Replicate 1 & 0.370 & 0.354 & 0.386 & 0.342 \\
\hline & Replicate 2 & 0.381 & 0.366 & 0.398 & 0.354 \\
\hline & Replicate 3 & 0.357 & 0.340 & 0.373 & 0.328 \\
\hline & Replicate 4 & 0.388 & 0.373 & 0.405 & 0.361 \\
\hline
\end{tabular}

Each row represents probe ratio distributions from replications of amplified DNA samples; each column represents probe ratio distributions from replications of unamplified DNA samples. The median values are 0.407 and 0.368 for REPLI-g and GenomiPhi, respectively. UnAmp (unamplified samples); Amp-R (REPLI-g amplified samples); Amp-G (GenomiPhi amplified samples). 
Table 3 Average CNV at various cut-off ratios

\begin{tabular}{llccccc}
\hline Sample & Comparison* & $\begin{array}{c}\text { Percentage } \\
\text { with ratios }>\text { 1.5 }\end{array}$ & $\begin{array}{c}\text { Percentage } \\
\text { with ratios }>\text { 2.0 }\end{array}$ & $\begin{array}{c}\text { Percentage } \\
\text { with ratios }>\mathbf{2 . 5}\end{array}$ & $\begin{array}{c}\text { Percentage } \\
\text { with ratios }>\mathbf{3 . 0}\end{array}$ & $\begin{array}{c}\text { Percentage } \\
\text { with ratios }>\text { 4.0 }\end{array}$ \\
\hline Normal & UnAmp/UnAmp & 0.004 & 0.000 & 0.000 & 0.000 & 0.000 \\
& Amp-R/UnAmp & 2.043 & 0.525 & 0.194 & 0.077 & 0.010 \\
& Amp-G/UnAmp & 8.139 & 1.273 & 0.339 & 0.109 & 0.017 \\
CF1 & Amp-R/UnAmp & 2.191 & 0.478 & 0.162 & 0.057 & 0.010 \\
& Amp-G/UnAmp & 3.682 & 0.747 & 0.230 & 0.074 & 0.015 \\
CF2 & Amp-R/UnAmp & 1.913 & 0.486 & 0.192 & 0.075 & 0.007 \\
& Amp-G/UnAmp & 2.883 & 0.605 & 0.183 & 0.061 & 0.015 \\
\hline
\end{tabular}

* Indicates samples on the CGH array. The averages of the 4 replicate WGA are given. UnAmp (unamplified samples); Amp-R (REPLI-g amplified samples);

Amp-G (GenomiPhi amplified samples).

corresponding native unamplified sample. The first plot shows the distribution of average $\log _{2}$ ratios of unamplified Normal DNA vs. unamplified Normal DNA (self-self hybridization). The distribution is centered at 0 and is very tight, indicating that little variability (i.e., $\mathrm{CNV}$ "noise") is introduced by the aCGH process itself. Supporting this conclusion, the derivative $\log _{2}$ ratio spread (which is the average log ratio difference between adjacent probes of an array) for the 4 replicate self-self hybridizations was low (0.165). Thus, any change in the distribution of $\log _{2}$ ratios in amplified samples compared to unamplified samples could be considered to be due primarily to the WGA process. A notably wider distribution of the ratios was observed for the amplified samples compared to the unamplified samples although the median was still close to 0 . These results suggest that $\mathrm{CGH}$ arrays can be sensitive tools for this type of $\mathrm{CNV}$ analysis. More importantly, WGA resulted in CNV changes for all three samples. The majority of the CNV changes were due to under-amplification.

To better understand differences in copy number introduced by the WGA process, we compared the

Table 4 Common highly under-amplified regions ( $>3$ fold) in Normal, CF1, and CF2 samples after WGA

\begin{tabular}{|c|c|c|c|c|c|}
\hline Chromosome & Cytoband & Stat (bp) & Stop (bp) & No. of Probes & No. of Genes* \\
\hline Chr1 & p36.33 - p36.32 & 787630 & 3693246 & 967 & 88 \\
\hline Chr2 & $\mathrm{q} 37.3$ & 241165907 & 241864699 & 258 & 13 \\
\hline Chr4 & p16.3 & 533101 & 3842102 & 668 & 38 \\
\hline Chr5 & p15.33 & 25942 & 2285711 & 724 & 30 \\
\hline Chr7 & $\mathrm{p} 22.3$ & 42776 & 2759847 & 734 & 30 \\
\hline Chr8 & q24.3 & 142067269 & 146261757 & 1480 & 110 \\
\hline Chr9 & q34.2 - q34.3 & 136872507 & 140734384 & 1500 & 106 \\
\hline Chr10 & p15.3 & 499391 & 681067 & 86 & 1 \\
\hline Chr11 & p15.5 - p15.4 & 271403 & 3216315 & 997 & 78 \\
\hline Chr12 & p13.33-p13.31 & 163393 & 7069315 & 87 & 6 \\
\hline Chr13 & q34 & 114305327 & 114924254 & 174 & 8 \\
\hline Chr14 & q32.31-p32.32 & 101469473 & 105943028 & 741 & 63 \\
\hline Chr16 & p13.3 & 93428 & 3286713 & 1256 & 169 \\
\hline Chr16 & $q 24.2-q 24.3$ & 87871999 & 89999459 & 555 & 35 \\
\hline Chr17 & p13.3 & 47346 & 1964509 & 814 & 13 \\
\hline Chr17 & q25.2-p25.3 & 74886218 & 80217558 & 601 & 62 \\
\hline Chr18 & q23 & 77064970 & 77617915 & 219 & 6 \\
\hline Chr19 & p13.3 & 259195 & 2442313 & 983 & 103 \\
\hline Chr20 & q13.33 & 60782793 & 62819250 & 880 & 91 \\
\hline ChrX & $\mathrm{q} 28$ & 152713107 & 153194997 & 218 & 23 \\
\hline ChrY & p11.32 & 10891 & 1663423 & 443 & 19 \\
\hline
\end{tabular}

Cytobands and chromosomal locations are based on hg 19 (NCBI Genome build 37).

* total number of genes located within the under-amplified regions. 
Table 5 Pair-wise comparison of Kolmogorov distances between probe ratio distributions within each sample group

\begin{tabular}{|c|c|c|c|c|c|c|}
\hline \multirow[t]{2}{*}{ Sample* } & & \multicolumn{5}{|c|}{ Kolmogorov distance } \\
\hline & & Replicate 1 & Replicate 2 & Replicate 3 & & Replicate 4 \\
\hline Normal & Replicate 1 & 0 & & & 0.025 & \\
\hline \multirow[t]{3}{*}{ UnAmp/UnAmp } & Replicate 2 & 0.019 & 0 & & & \\
\hline & Replicate 3 & 0.026 & 0.044 & 0 & & \\
\hline & Replicate 4 & 0.024 & 0.018 & 0.049 & & 0 \\
\hline Normal & Replicate 1 & 0 & & & 0.013 & \\
\hline \multirow[t]{3}{*}{ Amp-R/UnAmp } & Replicate 2 & 0.009 & 0 & & & \\
\hline & Replicate 3 & 0.011 & 0.005 & 0 & & \\
\hline & Replicate 4 & 0.015 & 0.020 & 0.021 & & 0 \\
\hline Normal & Replicate 1 & 0 & & & 0.029 & \\
\hline \multirow[t]{3}{*}{ Amp-G/UnAmp } & Replicate 2 & 0.026 & 0 & & & \\
\hline & Replicate 3 & 0.020 & 0.033 & 0 & & \\
\hline & Replicate 4 & 0.048 & 0.023 & 0.053 & & 0 \\
\hline CF1 & Replicate 1 & 0 & & & 0.085 & \\
\hline \multirow[t]{3}{*}{ Amp-R/UnAmp } & Replicate 2 & 0.108 & 0 & & & \\
\hline & Replicate 3 & 0.046 & 0.062 & 0 & & \\
\hline & Replicate 4 & 0.065 & 0.062 & 0.120 & & 0 \\
\hline CF1 & Replicate 1 & 0 & & & 0.053 & \\
\hline \multirow[t]{3}{*}{ Amp-G/UnAmp } & Replicate 2 & 0.050 & 0 & & & \\
\hline & Replicate 3 & 0.056 & 0.023 & 0 & & \\
\hline & Replicate 4 & 0.042 & 0.082 & 0.095 & & 0 \\
\hline CF2 & Replicate 1 & 0 & & & 0.047 & \\
\hline \multirow[t]{3}{*}{ Amp-R/UnAmp } & Replicate 2 & 0.003 & 0 & & & \\
\hline & Replicate 3 & 0.065 & 0.066 & 0 & & \\
\hline & Replicate 4 & 0.029 & 0.029 & 0.088 & & 0 \\
\hline CF2 & Replicate 1 & 0 & & & 0.039 & \\
\hline \multirow[t]{3}{*}{ Amp-G/UnAmp } & Replicate 2 & 0.018 & 0 & & & \\
\hline & Replicate 3 & 0.050 & 0.036 & 0 & & \\
\hline & Replicate 4 & 0.055 & 0.043 & 0.009 & & 0 \\
\hline
\end{tabular}

* Indicates samples on CGH array.

The median values for each group are shown in bold. UnAmp (unamplified samples); Amp-R (REPLI-g amplified samples); Amp-G (GenomiPhi amplified samples).

$\log _{2}$ ratio distribution of the Normal unamplified DNA vs. Normal unamplified DNA (self-self) with the $\log _{2}$ ratio distribution of the WGA Normal (amplified) DNA vs. Normal unamplified DNA by calculating the Kolmogorov distances. The pair-wise comparisons for both the REPLI-g and GenomiPhi WGA methods are shown in Table 2. The median pair-wise Kolmogorov distances between the REPLIg-amplified vs. unamplified distribution and GenomiPhi-amplified vs. unamplified distribution are 0.407 and 0.368 , respectively, consistent with differences between the distributions of $\log _{2}$ ratios before and after WGA.

Another measure of the uniformity of the WGA process is the percentage of probes with ratios below or above a certain cut-off value when the WGA DNA is compared to the unamplified DNA. Table 3 shows the percentage of probes on the $\mathrm{CGH}$ arrays with ratios greater than 1.5-fold, 2-fold, 2.5-fold, 3-fold, and 4-fold for the three DNA samples and the two WGA methods. The percentages are the average of the 4 replicates. Also shown is the percentage of probes above these cut-offs for the unamplified Normal DNA compared to itself; all ratios were within 2 , consistent with low variability of the aCGH method. Both amplification methods resulted in less than $1 \%$ of probes showing $\mathrm{CNV}$ of greater than 2-fold, with the exception of the Normal sample amplified by GenomiPhi (1.273\%). If the fold-change criterion was relaxed to 3 -fold, less than approximately $0.1 \%$ of probes showed CNV. Table 4 shows regions of the 
Table 6 Pair-wise comparison of Phi correlations of probe ratios greater than 2 within each sample group after CBS smoothing

\begin{tabular}{|c|c|c|c|c|c|c|}
\hline \multirow[t]{2}{*}{ Sample* } & & \multicolumn{5}{|c|}{ Phi correlation value } \\
\hline & & Replicate 1 & Replicate 2 & Replicate 3 & & Replicate 4 \\
\hline Normal & Replicate 1 & 1 & & & 1.000 & \\
\hline \multirow[t]{3}{*}{ UnAmp/UnAmp } & Replicate 2 & 1.000 & 1 & & & \\
\hline & Replicate 3 & 1.000 & 1.000 & 1 & & \\
\hline & Replicate 4 & 1.000 & 1.000 & 1.000 & & 1.000 \\
\hline Normal & Replicate 1 & 1 & & & 0.842 & \\
\hline \multirow[t]{3}{*}{ Amp-R/UnAmp } & Replicate 2 & 0.855 & 1 & & & \\
\hline & Replicate 3 & 0.840 & 0.843 & 1 & & \\
\hline & Replicate 4 & 0.877 & 0.840 & 0.809 & & 1 \\
\hline Normal & Replicate 1 & 1 & & & 0.805 & \\
\hline \multirow[t]{3}{*}{ Amp-G/UnAmp } & Replicate 2 & 0.853 & 1 & & & \\
\hline & Replicate 3 & 0.839 & 0.796 & 1 & & \\
\hline & Replicate 4 & 0.773 & 0.814 & 0.707 & & 1 \\
\hline CF1 & Replicate 1 & 1 & & & 0.611 & \\
\hline \multirow[t]{3}{*}{ Amp-R/UnAmp } & Replicate 2 & 0.597 & 1 & & & \\
\hline & Replicate 3 & 0.764 & 0.665 & 1 & & \\
\hline & Replicate 4 & 0.457 & 0.625 & 0.520 & & 1 \\
\hline \multirow{4}{*}{$\begin{array}{l}\text { CF1 } \\
\text { Amp-G/UnAmp }\end{array}$} & Replicate 1 & 1 & & & 0.667 & \\
\hline & Replicate 2 & 0.687 & 1 & & & \\
\hline & Replicate 3 & 0.679 & 0.777 & 1 & & \\
\hline & Replicate 4 & 0.632 & 0.656 & 0.589 & & 1 \\
\hline CF2 & Replicate 1 & 1 & & & 0.768 & \\
\hline \multirow[t]{3}{*}{ Amp-R/UnAmp } & Replicate 2 & 0.824 & 1 & & & \\
\hline & Replicate 3 & 0.697 & 0.744 & 1 & & \\
\hline & Replicate 4 & 0.796 & 0.793 & 0.707 & & 1 \\
\hline CF2 & Replicate 1 & 1 & & & 0.780 & \\
\hline \multirow[t]{3}{*}{ Amp-G/UnAmp } & Replicate 2 & 0.865 & 1 & & & \\
\hline & Replicate 3 & 0.798 & 0.746 & 1 & & \\
\hline & Replicate 4 & 0.762 & 0.728 & 0.838 & & 1 \\
\hline
\end{tabular}

* Indicates samples on CGH array.

The median Phi correlation values from top to bottom are shown in bold. UnAmp (unamplified samples); Amp-R (REPLI-g amplified samples); Amp-G (GenomiPhi amplified samples).

genome that were consistently under-amplified by at least 3-fold across all samples by both amplification methods. These under-amplified regions represent a small portion of total genome (2.1\%), and contain about 1099 genes (approximately 4.4\%). Thus, while the MDA technology resulted in changes in copy number, the changes were relatively small in most regions of the genome, and were concentrated in a relatively small number of chromosomal loci.

\section{Consistency of the WGA process}

Differences in the distribution of ratios within each sample group were calculated using the Kolmogorov distance. Table 5 shows pair-wise comparisons of Kolmogorov distances within each sample group, as well as the median value for each group. The median Kolmogorov value for the four self-self hybridizations (Normal sample, unamplified vs. unamplified) was 0.025 and the median Kolmogorov values within the other sample groups were similarly low. Thus, the WGA process appears to be reproducible in terms of uniformity of amplification throughout the genome. The Phi correlation was calculated to evaluate the consistency of the position of the CNV loci (defined as having a ratio of greater than 2). Higher values (greater than 0.7) indicate the ratios at each probe location are very similar. Results 
Table 7 Pair-wise comparison of Kolmogorov distances of distribution ratios between the REPLI-g and GenomiPhi methods

\begin{tabular}{|c|c|c|c|c|c|}
\hline \multirow[t]{3}{*}{ Sample } & & \multicolumn{4}{|c|}{ Kolmogorov distance } \\
\hline & & \multicolumn{4}{|c|}{ Amp-G/UnAmp } \\
\hline & & Replicate 1 & Replicate 2 & Replicate 3 & Replicate 4 \\
\hline Normal & Replicate 1 & 0.031 & 0.055 & 0.026 & 0.078 \\
\hline \multirow[t]{3}{*}{ Amp-R/UnAmp } & Replicate 2 & 0.038 & 0.063 & 0.035 & 0.085 \\
\hline & Replicate 3 & 0.041 & 0.065 & 0.037 & 0.088 \\
\hline & Replicate 4 & 0.032 & 0.051 & 0.019 & 0.072 \\
\hline CF1 & Replicate 1 & 0.095 & 0.050 & 0.041 & 0.131 \\
\hline \multirow[t]{3}{*}{ Amp-R/UnAmp } & Replicate 2 & 0.017 & 0.061 & 0.071 & 0.025 \\
\hline & Replicate 3 & 0.050 & 0.009 & 0.019 & 0.085 \\
\hline & Replicate 4 & 0.079 & 0.115 & 0.130 & 0.037 \\
\hline CF2 & Replicate 1 & 0.082 & 0.069 & 0.033 & 0.027 \\
\hline \multirow[t]{3}{*}{ Amp-R/UnAmp } & Replicate 2 & 0.083 & 0.069 & 0.034 & 0.028 \\
\hline & Replicate 3 & 0.021 & 0.019 & 0.034 & 0.039 \\
\hline & Replicate 4 & 0.106 & 0.095 & 0.060 & 0.052 \\
\hline
\end{tabular}

The median values for Normal, CF1, and CF2 groups between the WGA methods are 0.046, 0.055, and 0.046, respectively. Amp-R (REPLI-g amplified); Amp-G (GenomiPhi amplified).

of pair-wise Phi correlations (Table 6) indicate that the majority of the replicates within each sample/amplification group are highly correlated (similar patterns of CNVs). The exception is the CF1 sample, which has a Phi correlation just below 0.7. The Phi correlation values are above 0.7 if the fold cut-off is set at 2.5 (data not shown). Overall, the results from the Kolmogorov distances and Phi correlations show that there is high consistency in the WGA process within each sample/ WGA method group.
Comparisons between the two whole genome amplification kits

Both WGA kits used in this study, REPLI-g and GenomiPhi, use the same bacterial phage Ф29 DNA polymerase. However, the assay procedures are considerably different with regard to reaction time $(4 \mathrm{hr}$ with GenomiPhi and $10 \mathrm{hr}$ with REPLI-g) and method of DNA denaturation (heat with GenomiPhi and alkaline with REPLI-g). Thus, comparison of outcomes of these procedures is important. The median values of pair-wise

Table 8 Pair-wise comparisons of Phi correlations of probe ratios greater than 2 between GenomiPhi and REPLI-g amplified samples

\begin{tabular}{|c|c|c|c|c|c|}
\hline \multirow[t]{3}{*}{ Sample } & & \multicolumn{4}{|c|}{ Phi correlation value } \\
\hline & & \multicolumn{4}{|c|}{ Amp-G/UnAmp } \\
\hline & & Replicate 1 & Replicate 2 & Replicate 3 & Replicate 4 \\
\hline Normal & Replicate 1 & 0.746 & 0.724 & 0.782 & 0.725 \\
\hline \multirow[t]{3}{*}{ Amp-R/UnAmp } & Replicate 2 & 0.729 & 0.712 & 0.783 & 0.706 \\
\hline & Replicate 3 & 0.688 & 0.664 & 0.751 & 0.653 \\
\hline & Replicate 4 & 0.814 & 0.772 & 0.811 & 0.763 \\
\hline CF1 & Replicate 1 & 0.736 & 0.804 & 0.906 & 0.681 \\
\hline \multirow[t]{3}{*}{ Amp-R/UnAmp } & Replicate 2 & 0.842 & 0.782 & 0.711 & 0.807 \\
\hline & Replicate 3 & 0.828 & 0.804 & 0.815 & 0.738 \\
\hline & Replicate 4 & 0.733 & 0.692 & 0.622 & 0.796 \\
\hline CF2 & Replicate 1 & 0.762 & 0.771 & 0.833 & 0.835 \\
\hline \multirow[t]{3}{*}{ Amp-R/UnAmp } & Replicate 2 & 0.776 & 0.773 & 0.831 & 0.818 \\
\hline & Replicate 3 & 0.789 & 0.839 & 0.782 & 0.795 \\
\hline & Replicate 4 & 0.736 & 0.741 & 0.825 & 0.828 \\
\hline
\end{tabular}

The median values for Normal, CF1, and CF2 groups were $0.738,0.789$, and 0.792 , respectively. 


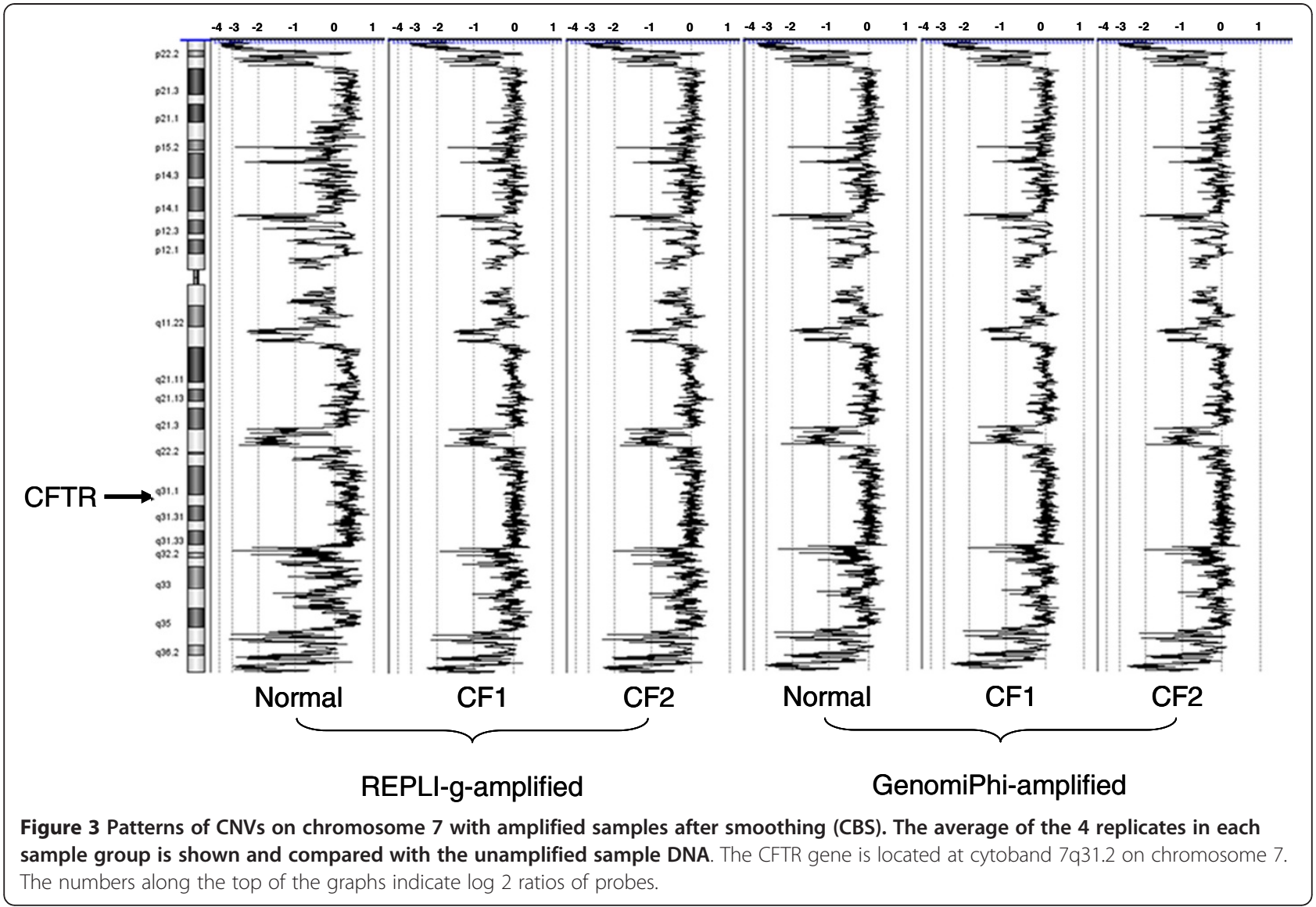

Kolmogorov distances for Normal, CF1, and CF2 samples between REPLI-g and GenomiPhi amplified samples are $0.046,0.055$, and 0.046 , respectively (Table 7 ) which are similar to the Kolmogorov distances from samples amplified by the same method (range: 0.013- 0.085; see Table 4). The median pair-wise Phi correlations for Normal, CF1, and CF2 samples between REPLI-g and GenomiPhi amplified samples after CBS smoothing are 0.739, 0.789 and 0.792 , respectively (Table 8). Again, these values are similar to those obtained when comparing within an amplification method (see Table 6). These results indicate that the two amplification methods generate similar amplified DNA samples despite the procedural differences.

Visual comparison of CNV generated by these amplification methods across chromosome 7 is illustrated in Figure 3. The pattern of CNVs is similar between the 2 amplification methods and among the 3 DNA

Table 9 Probe information for TaqMan Copy Number Quantitative PCR

\begin{tabular}{llll}
\hline PCR Probe & Applied Biosystems probe ID & CFTR location & Chromosome location (Approx.) \\
\hline I & Hs05020079_cn & Intron 1 & Chr7: 117122192 \\
II & Hs04952703_cn & Intron 2 & Chr7: 117145581 \\
III & Hs04988506_cn & Intron 3 & Chr7: 117153814 \\
IV & Hs04984230_cn & Intron 9 & Chr7: 117185643 \\
V & Hs04963787_cn & Intron 11 & Chr7: 117203673 \\
VI & Hs05017940_cn & Intron 12 & Chr7: 117228242 \\
VII & Hs05001680_cn & Intron 15 & Chr7: 117238610 \\
VIII & Hs04963453_cn & Intron 18 & Chr7: 117247359 \\
IX & Hs04947556_cn & Intron 23 & Chr7: 117282984 \\
$X$ & Hs00393982_cn & Intron 27 & Chr7: 117307221 \\
\hline
\end{tabular}

Chromosome locations are based on NCBI Human Genome Build 37. 


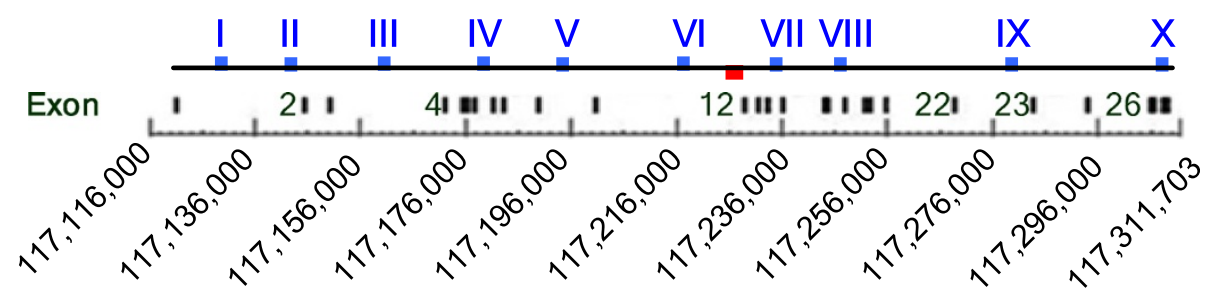

Figure 4 A schematic diagram of the PCR probe locations and a 2-kb sequencing region of the CFTR gene on chromosome 7. The blue boxes show the locations of the PCR probes (see Table 8). The small red box highlights the 2 kb region of the CFTR gene that was sequenced. This DNA segment includes part of intron 11 and exon 12, which account for $1 \%$ of the gene.

specimens, with much of the chromosome showing $\mathrm{CNV}<2$-fold when compared to the unamplified DNA. However, regions with consistent $>2$-fold CNV are evident near the chromosomal ends, as well as near 7q21.3, $7 q 11.22$, and 7p14.1, among others. The region near the CFTR gene appears to amplify uniformly and contains no CNVs greater than 2-fold.

\section{Quantitative real-time PCR copy number assay within the CFTR gene region}

Subsequent to array analysis, a quantitative PCR method was used to further evaluate the variability between the amplified and unamplified DNA samples. Ten TaqMan Copy Number PCR probes were selected from Applied Biosystem's pre-designed research assays across the length of the CFTR gene, a well-studied genetic disease locus, representing 9 introns and 1 exon (Table 9 and Figure 4). Relative copy number was calculated for each probe in reference to the unamplified DNA sample for each subject. Each of the ten probes gave comparable results showing less than 2 -fold difference (considering both over- and under-amplification) in fold-change between amplified and unamplified DNA samples (Figure 5). This low level of variability was also evident when comparing results between the two amplification methods
(REPLI-g and GenomiPhi), suggesting comparable results irrespective of the manufacturer's protocol. Furthermore, the amount of variability across the three human samples was similar, suggesting robust amplification results regardless of possible biological differences. Thus, the TaqMan Copy Number PCR results are in agreement with the aCGH data (Figure 3) in identifying low levels (below 2-fold change) of amplification variability introduced by the WGA process in the CFTR locus.

\section{DNA sequence analysis in the CFTR gene region}

Bidirectional Sanger DNA sequencing was performed on an approximately $2 \mathrm{~kb}$ region of the CFTR gene to examine the fidelity of the WGA process using primers shown in Table 10 (see Methods). DNA sequences from the WGA samples were compared with the respective unamplified DNA samples. The results are shown in Table 11 and there were no detectable mutations introduced by either WGA method in the 3 DNA samples (approximate 7,900 nucleotides examined for each sample/WGA method). Thus, the level of detectable mutations introduced by the GenomiPhi and REPLI-g WGA methods in this analysis was less than 1 mutation per 23,000 nucleotides examined. These results suggest that both GenomiPhi and REPLI-g

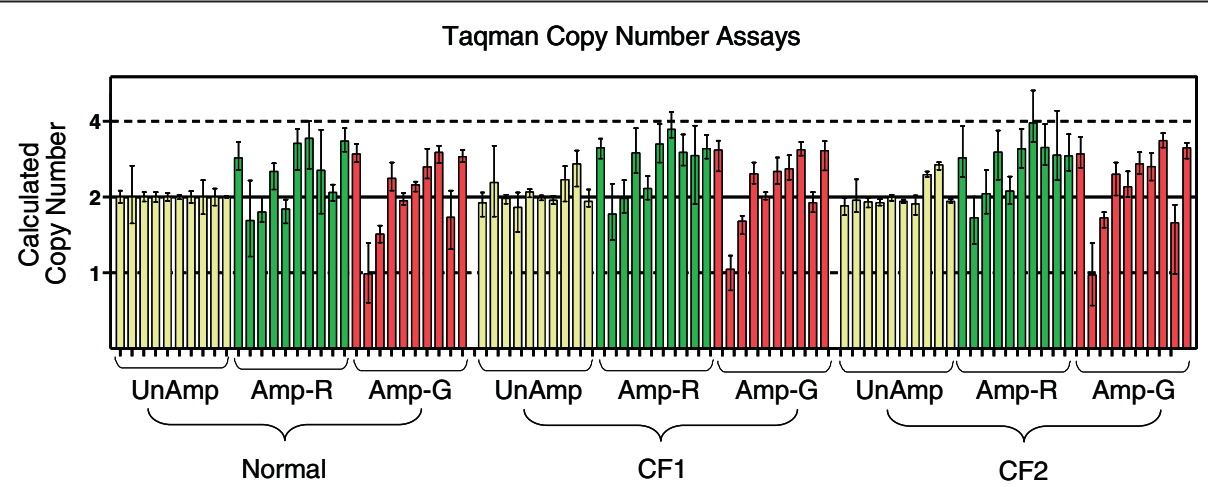

Figure 5 Quantitative real time PCR copy number assays. Taqman assays were used to examine copy number variations at 10 locations within the CFTR gene in both the unamplified and amplified DNA samples. Average calculated copy number values are plotted with bars representing minimum and maximum values from replicate measurements $(n=4)$. Within each amplification / sample group, the order of the probe results is I- X (see Figure 4). UnAmp (no amplification); Amp-R (REPLI-g amplified); Amp-G (GenomiPhi amplified). 
Table 10 Sequencing primer information

\begin{tabular}{llclc}
\hline Primer Name & Primer location & Primer sequence $\mathbf{( 5}^{\prime}$ - $\mathbf{3}^{\prime}$ ) & Tm & Amplicon size (bp) \\
\hline Seq1_F & $112776-112797$ & GGACATCTCCAAGTTTGCAGAG & 66 & 760 \\
Seq1_R & $113535-113512$ & GAAACATTGACATCAGAGTCAC & 64 & 729 \\
Seq2_F & $113459-113479$ & GTCAAGGAGAGAGCTTTGTGG & 64 & 756 \\
Seq2_R & $114187-114165$ & TGGACAACACATTACACATTCTG & 64 \\
Seq3_F & $114050-114071$ & GGCTTCTAGACATCCAACATAG & 64 & 756 \\
Seq3_R & $114805-114784$ & GATAGCAGTGCTGCCACAACTG & 68 & \\
\hline
\end{tabular}

Primer locations (chromsome 7) are based on NCBI reference sequence NG_016465.1. "F" -forward primer; "R"- Reverse primer.

appear to be robust and accurate methods for amplifying nanogram quantities of starting DNAs to microgram levels.

\section{Discussion}

The sequencing of the human genome, coupled with advances in genomics technologies, is having a major impact on understanding basic human biology, as well as the molecular causes of diseases and toxicities [38]. In fact, the leaders of the U.S. Food and Drug Administration (FDA) and the National Institutes of Health (NIH) have recently outlined the opportunities in clinical medicine that are being created by advances in basic science, including genomics [39]. The two agencies have announced a new collaborative effort to focus regulatory and translational sciences on bringing medical products and therapies into the age of personalized medicine. This growing store of knowledge has enormous potential application to the development of devices and tests for use in the diagnosis, mitigation, treatment, cure, and prevention of disease and other conditions.

As of 2010, more than 100,000 germline mutations in more than 3700 genes have been associated with human inherited disease, with about 300 new disease genes and 10,000 mutations being identified annually [40]. In principle, DNA tests could be developed for each of these conditions. Development and approval of such tests for clinical diagnostics often require that accuracy be demonstrated on patient samples. That is, the test needs to detect the disease-causing mutation with high precision and accuracy. In addition, measures of proficiency are required by testing laboratories to ensure continued accuracy of the results. The Clinical Laboratory Improvement Advisory Committee has recently issued a good practices report for molecular genetic testing and has stressed the need for performance assessments [41]. Such recommendations are supportive of the 2008 Report of the Secretary's Advisory Committee on Genetics, Health, and Society (SACGHS) [42]. Additionally, the SACGHS has identified the development of genetic reference materials as one of five critical gaps in the oversight system of genetic testing [42]. The device approval process, use of reference and control materials, and ongoing proficiency assessments require relatively large quantities of clinical samples in order to assure test performance is adequate and maintained over time. The availability of sufficient samples with appropriate mutations has been recognized as a critical issue in genetic testing, given the paucity of validated clinical inherited disease gene samples [43]. Therefore, methods that can expand the limited supply of validated clinical samples would have major impacts on both the test and device performance evaluation and approval process, and the on-going proficiency assessment of the test providers.

The importance of the amount of template DNA for MDA-based WGA has been thoroughly discussed for SNP assays and STR genotyping in previous studies $[28,31]$. For example, large number of SNPs could be accurately detected from as low as $0.01 \mathrm{ng}$ of DNA template $[27,28]$ and even degraded DNA samples can be used for forensic SNP typing [29], while over $100 \mathrm{ng}$

Table 11 Mutations in the WGA DNA

\begin{tabular}{|c|c|c|c|c|}
\hline \multirow[t]{2}{*}{ Sample } & \multirow[t]{2}{*}{ Amplification method } & \multirow[t]{2}{*}{ Nucleotides examined } & \multicolumn{2}{|c|}{ Mutation } \\
\hline & & & Total number & Percentage \\
\hline Normal & REPLI-g & 7900 & 0 & $<0.01$ \\
\hline CF1 & REPLI-g & 7924 & 0 & $<0.01$ \\
\hline CF2 & REPLI-g & 7909 & 0 & $<0.01$ \\
\hline Normal & GenomiPhi & 7888 & 0 & $<0.01$ \\
\hline CF1 & GenomiPhi & 7905 & 0 & $<0.01$ \\
\hline CF2 & GenomiPhi & 7906 & 0 & $<0.01$ \\
\hline
\end{tabular}

Nucleotides examined are the sum of $2-\mathrm{kb}$ sequences from the four replications. 
of DNA template was needed for optimal STR genotyping [31]. In this study, we mainly focused on evaluating the uniformity and fidelity of WGA DNAs using CGH arrays, TaqMan copy number assays, and DNA sequencing. The results indicated the amplified DNA and its native unamplified DNA that we examined were similar, although not identical, in terms of DNA copy number variation. Importantly, no detectable introduced mutations were found under our experimental conditions.

The Agilent Human CGH array used in this study includes approximately 970,000 probes at 1 to $2 \mathrm{~kb}$ intervals throughout the human genome. To ensure the quality of this study, four independent replications for both the WGA and aCGH processes were used. The self-self hybridization of unamplified Normal DNA samples provided us the base-line measurement of noise for aCGH technology. The box plot of unamplified Normal samples indicated less than $0.004 \%$ of the approximately 970,000 probes on the $\mathrm{CGH}$ array have fold changes greater than 1.5 and no CNVs can be detected by aCGH (Figure 2 and Table 3). The low Kolmogorov distances (0.025) and high Phi correlations (1.00) between the four replicates of unamplified samples further showed the aCGH technology was reproducible and could be a reliable tool to access variability of the WGA process.

The WGA technologies have evolved over the years from Taq DNA polymerase-based (PCR-based) to bacteriophage $\Phi 29$ DNA polymerase-based methods (MDA). We focused on MDA methods in this study because of the processivity and reportedly low replication error rate of the $\Phi 29$ DNA polymerase. In this study, we compared two MDA-based commercial WGA kits: the REPLI-g and GenomiPhi kits. The comparison between the unamplified and amplified DNA samples showed that relatively few probes (approximately $1 \%$ or less) were over- or under-amplified by more than 2-fold (Table 3). GenomiPhi-amplified DNA, however, showed consistently more CNVs than the REPLI-g-amplified DNA. Kolmogorov distances also indicated that differences in the distribution of DNA copy number existed between the unamplified and amplified samples (Table 2), and that these differences were greater than the differences between the REPLI-g and GenomiPhi amplified samples (Table 7). Thus, even though the kits differ in method of DNA denaturation (heat vs. alkaline), buffer composition, and reaction time ( $4 \mathrm{hr}$ vs. $10 \mathrm{hr}$ ), they produce similar amplified DNA, likely the result of the common $\Phi 29$ DNA polymerase.

The data in this report indicate that the two WGA methods examined can consistently amplify small amounts of DNA (ng) to large quantities $(\sim 40 \mu \mathrm{g})$ with relatively small changes in DNA copy number along the chromosomes. Changes in DNA copy number of greater than 3-fold are evident as under-amplified regions at the ends of chromosomes, as illustrated in Figure 3, and in discrete regions on many of the chromosomes (Table 4). Because of the consistency of the DNA amplification, knowledge of the positions of likely under-amplification can prevent the inappropriate use of WGA DNA. Results presented in Table 4 may serve as a reference guide to avoid target genes in these regions. Inspection of the GC content within these consistently under-amplified regions showed an average GC content of nearly 53\% which was higher than the overall GC content of the human genome of $41 \%$. Further investigations will be needed to evaluate the significance of this observation.

Cystic fibrosis (CF) is caused by severe dysfunction of cystic fibrosis transmembrane conductance regulator (CFTR), which commonly leads to progressive lung disease and a shortened life [44]. Currently, there is no cure available for CF, even though multiple interventions have been developed to slow its progression. Since the FDA cleared the first genotyping assay for CF in 2005, manufacturers have developed a number of genotyping tests for rare heritable diseases caused by multiple mutant alleles, using patient samples to assess performance. There is an increased interest in using WGA DNA samples created from patient samples to support the performance of the assays. This study shows that within certain limits, the WGA process produces large quantities of DNA that may be useful for this purpose. To address the concerns of mutations introduced by WGA, TaqMan copy number assays were used to analyze CNVs at 10 locations within CFTR gene region and a random selected $2 \mathrm{~kb}$ region in CFTR gene was also sequenced. Results from the TaqMan copy number assay are in excellent agreement with the aCGH results. The DNA sequencing analysis showed there were no mutations induced by WGA in this approximately $2 \mathrm{~kb}$ region of the CFTR gene (mutation induction was less than $4 \times 10^{-5}$ ), which indicates that WGA DNAs can be used for enrichment of DNA samples for cystic fibrosis genotyping assays.

The objective of the study presented here was to determine whether WGA amplified samples may be a reliable alternative to native clinical specimens for assessing the performance of a test under investigation. The conclusions from this study provide scientific input that may serve to support regulatory decisions in the ascertainment of safety and effectiveness of diagnostic products that use whole genome amplified samples in clinical studies. This study may serve as a guide to the technical qualification of WGA DNA for assessing the performance of genotyping assays.

\section{Conclusions}

In summary, WGA generates large quantities of DNA with relatively high uniformity and low replication error 
rate when compared to unamplified DNA. This suggests that WGA DNA may be suitable for accurate genotyping. However, because there are consistent differences between the WGA DNA and the native unamplified DNA, characterization of the genomic region of interest, as described here, will be necessary to ensure the reliability of genotyping results from WGA DNA.

\section{Methods}

DNA Samples: DNA samples used in this study were purchased from PrecisionMed, Inc. (San Diego, CA) and are shown in Table 1. All three DNA samples were extracted from human blood and were dissolved in TE buffer (1 mM EDTA, $10 \mathrm{mM}$ Tris at $\mathrm{pH}$ 8.0). The concentration and purity (A260/280 ratio) of chromosomal DNA was measured by the NanoDrop 1000 and PicoGreen methods (Life Technologies, Carlsbad, CA). DNA samples were aliquoted to four batches and stored at $-20{ }^{\circ} \mathrm{C}$ before use.

Whole Genome Amplification (WGA): Two WGA kits, GenomiPhi (GE Healthcare, Piscataway, NJ ) and REPLI-g (Qiagen, Inc., Hilden, Germany), were used in this study. $20 \mathrm{ng}$ of DNA template was used for the amplification process using the manufacturers' recommended protocols (Illustra GenomiPhi HY DNA Amplification kit protocol and REPLI-g Mini/Midi Handbook). The GenomiPhi reaction was allowed to proceed for $4 \mathrm{hr}$ while the REPLI-g reaction was allowed to proceed for $10 \mathrm{hr}$. Both kits use the same phage Ф29 DNA polymerase to amplify the DNA templates, although the buffer components are proprietary. The average yields after WGA with GenomiPhi and REPLI-g were $37.5 \mu \mathrm{g}$ and 14.0 $\mu$ g DNA, respectively.

Array Comparative Genome Hybridization (aCGH): The Agilent (Santa Clara, CA) 1 M human CGH array used in this study is based on NCBI Build 37 (UCSC) with 963,029 biological features and 6,685 controls. The majority of the probes on this array have 1 to $2 \mathrm{~kb}$ spacing along the human chromosomes. DNA labeling and hybridization were performed following the Agilent Oligonucleotide Array-Based CGH for Genomic DNA Analysis protocol (V 6.1, 2009). One $\mu \mathrm{g}$ of genomic DNAs were labeled with fluorescent dyes (Cy3 or Cy5dUTP). In this study, the amplified DNA samples were labeled with Cy3 and unamplified DNA samples were labeled with Cy5. Labeled DNAs with specific activity greater than $20 \mathrm{pmol}$ of dye/ $\mathrm{\mu g}$ DNA were used for aCGH. Labeled amplified (Cy3) and unamplified (Cy5) DNA samples were paired and co-hybridized to the arrays at $65{ }^{\circ} \mathrm{C}$ for $40 \mathrm{hrs}$, then washed at room temperature following the Agilent Oligonucleotide Array-Based CGH for Genomic DNA Analysis protocol (V 6.1, 2009). The hybridized array was immediately scanned with an Agilent DNA Microarray Scanner (Agilent Technologies, Inc.) at $2 \mu \mathrm{m}$ resolution. The resulting images were analyzed by quantifying the $\mathrm{Cy} 3$ and Cy5 fluorescence intensity at each feature on the array using the Agilent Feature Extraction Software (V10.5). The fluorescence intensity of each pixel within the feature was determined and the median fluorescence of these pixel measurements was taken as the measure of fluorescence for the whole feature after subtraction of background. Dye bias was removed by linear normalization using the Agilent Feature Extraction Software before the intensity values were used to calculate ratios at each feature.

aCGH Data Analysis: Copy number variation (CNV) was calculated at each locus along each chromosome as the ratio of the sample (either unamplified or amplified) to the unamplified sample intensities. A modified algorithm [45] of the circular binary segmentation smoothing method (CBS, [46]) was used to partition these ratio measurements into chromosomal regions containing loci with equal copy numbers. Regions with ratios less than 2 were then defined as being unchanged (no CNV). Two approaches were used to measure the similarity of $\mathrm{CNV}$ patterns. To measure the difference between the distributions of ratios from different experimental groups, Kolmogorov distances were calculated which are the sub-distances between the distribution functions [47]. Low values indicate little difference between distributions. Phi correlation, which is a measure of association for two binary variables [48], was calculated to measure the consistency of locations of the CNVs. A low Kolmogorov distance coupled with a high Phi correlation indicates highly uniform amplification. Agilent Genomic Workbench 6.0 software (Agilent Technologies, Inc.) was also used to analyze CNVs along the chromosomes utilizing the CBS module. Raw data were imported into Agilent Genomic Workbench 6.0 for $\mathrm{CNV}$ analysis. The four replicates for each sample were combined based on the weight of each sample which is proportional to its quality in Agilent Genomic Workbench before analysis. Average aberration reports (minimal 3 consecutive probes within each section) for Normal, CF1, and CF2 after WGA were generated using the CBS method with a fold change cut-off at 3-fold. Cytobands with aberrations greater than 3 fold across the six amplified samples are summarized in Table 4.

Quantitative Real Time PCR Copy Number Assays: Unamplified and amplified genomic DNAs (10 ng) were used as templates in TaqMan Copy Number Assays (Applied Biosystems, Carlsbad, CA) according to the manufacturer's protocol. Ten probes, distributed across the length of the CFTR gene as illustrated in Figure 4, were used to quantify copy number in each DNA sample using RNase P as the reference assay. The Applied Biosystems probe IDs and locations in the CFTR gene can be found in Table 9. Cycle threshold (Ct) values were exported into Applied 
Biosystems CopyCaller Software to calculate the copy number at each locus. Copy number values were standardized to that of the unamplified DNA sample, according to software recommendation. Average relative copy number values were plotted along with minimum and maximum values of replicate measurements $(n=4)$.

DNA Sequencing Analysis: Unamplified and WGA DNAs were sent to SeqWright, Inc. (Houston, TX) for determination of the DNA sequence of a $2 \mathrm{~kb}$ region within the CFTR gene. The $2 \mathrm{~kb}$ region was PCR-amplified as 3 overlapping segments of $760 \mathrm{bp}, 729 \mathrm{bp}$, and $756 \mathrm{bp}$ as shown in Table 10 and Figure 4. The DNAs for sequencing included the 4 replicate WGA samples for each WGA method/DNA sample group (24 samples) plus one sample of each of the unamplified DNA samples. The DNA sequence of these amplicons was determined by bidirectional fluorescent dye-terminator chemistry using an ABI Prism 3730xl DNA sequencer. The sequencing data were aligned using Sequencher software (Gene Code Corporation, Ann Arbor, MI), which is based on an optimized Smith-Waterman algorithm. Nucleotide positions in which no base call was made or in which the forward base call was different from the reverse base call were excluded from further analysis. Of 23,744 bases examined after each amplification method, 53 bases $(0.2 \%)$ and 11 bases $(0.05 \%)$ were excluded after amplification with GenomiPhi and REPLI-g, respectively.

\section{Competing interests}

The authors declare that they have no competing interests.

\section{Acknowledgements}

The authors thank Drs. Elizabeth Mansfield, Tucker Patterson, and Nan Mei for valuable and constructive comments. We thank Dr. Yijun Ding for examination of the GC content of the under-amplified regions.

\section{Disclaimer}

The findings and conclusions in this report are those of the authors and do not necessarily represent the views of the U.S. FDA.

\section{Author details}

'Division of Systems Biology, National Center for Toxicological Research, FDA, Jefferson, AR 72079, USA. 2Division of Personalized Nutrition and Medicine, National Center for Toxicological Research, FDA, Jefferson, AR 72079, USA.

${ }^{3}$ Division of Genetic and Molecular Toxicology, National Center for Toxicological Research, FDA, Jefferson, AR 72079, USA. ${ }^{4}$ Office of In Vitro Diagnostic Device Evaluation \& Safety, Center for Devices and Radiological Health, FDA, Silver Spring, MD 20993, USA.

\section{Authors' contributions}

$J C F, T H, F M M, D R, Z T, R P$, and KB devised the project. TH and JCF were responsible for experimental design, data interpretation and writing the manuscript. CWC performed the statistical analysis. TH performed the aCGH analysis. JCK did the TaqMan copy number assay. YC and YG did sequencing alignment analysis. All authors read and approved the final manuscript.

Received: 20 January 2012 Accepted: 1 June 2012

Published: 1 June 2012

\section{References}

1. Montgomery GW, Campbell MJ, Dickson P, Herbert S, Siemering K, EwenWhite KR, Visscher PM, Martin NG: Estimation of the rate of SNP genotyping errors from DNA extracted from different tissues. Twin Res Hum Genet 2005, 8(4):346-352.

2. Zhou X, Temam S, Chen Z, Ye H, Mao L, Wong DT: Allelic imbalance analysis of oral tongue squamous cell carcinoma by high-density single nucleotide polymorphism arrays using whole-genome amplified DNA. Hum Genet 2005, 118(3-4):504-507.

3. Berthier-Schaad Y, Kao WH, Coresh J, Zhang L, Ingersoll RG, Stephens R, Smith MW: Reliability of high-throughput genotyping of whole genome amplified DNA in SNP genotyping studies. Electrophoresis 2007, 28(16):2812-2817.

4. Ng G, Roberts I, Coleman N: Evaluation of 3 methods of whole-genome amplification for subsequent metaphase comparative genomic hybridization. Diagn Mol Pathol 2005, 14(4):203-212.

5. Spits C, Le Caignec C, De Rycke M, Van Haute L, Van Steirteghem A, Liebaers I, Sermon K: Optimization and evaluation of single-cell wholegenome multiple displacement amplification. Hum Mutat 2006, 27(5):496-503.

6. Rook MS, Delach SM, Deyneko G, Worlock A, Wolfe JL: Whole genome amplification of DNA from laser capture-microdissected tissue for highthroughput single nucleotide polymorphism and short tandem repeat genotyping. Am J Pathol 2004, 164(1):23-33.

7. Hosono S, Faruqi AF, Dean FB, Du Y, Sun Z, Wu X, Du J, Kingsmore SF, Egholm M, Lasken RS: Unbiased whole-genome amplification directly from clinical samples. Genome Res 2003, 13(5):954-964.

8. Dietmaier W, Hartmann A, Wallinger S, Heinmoller E, Kerner T, Endl E, Jauch KW, Hofstadter F, Ruschoff J: Multiple mutation analyses in single tumor cells with improved whole genome amplification. Am J Pathol 1999, 154(1):83-95.

9. Telenius H, Carter NP, Bebb CE, Nordenskjold M, Ponder BA, Tunnacliffe A: Degenerate oligonucleotide-primed PCR: general amplification of target DNA by a single degenerate primer. Genomics 1992, 13(3):718-725.

10. Zhang L, Cui X, Schmitt K, Hubert R, Navidi W, Arnheim N: Whole genome amplification from a single cell: implications for genetic analysis. Proc Natl Acad Sci U S A 1992, 89(13):5847-5851.

11. Himmelbauer H, Schalkwyk LC, Lehrach H: Interspersed repetitive sequence (IRS)-PCR for typing of whole genome radiation hybrid panels. Nucleic Acids Res 2000, 28(2):e7.

12. Vos $P$, Hogers $R$, Bleeker M, Reijans M, van de Lee T, Hornes M, Frijters A, Pot J, Peleman J, Kuiper M, et al: AFLP: a new technique for DNA fingerprinting. Nucleic Acids Res 1995, 23(21):4407-4414.

13. Silander K, Saarela J: Whole genome amplification with Phi29 DNA polymerase to enable genetic or genomic analysis of samples of low DNA yield. Methods Mol Biol 2008, 439:1-18.

14. Dean FB, Hosono S, Fang L, Wu X, Faruqi AF, Bray-Ward P, Sun Z, Zong Q, Du Y, Du J, et al: Comprehensive human genome amplification using multiple displacement amplification. Proc Natl Acad Sci U S A 2002, 99 (8):5261-5266.

15. Blanco L, Bernad A, Lazaro JM, Martin G, Garmendia C, Salas M: Highly efficient DNA synthesis by the phage phi 29 DNA polymerase. Symmetrical mode of DNA replication. I Biol Chem 1989, 264(15):8935-8940

16. Lasken RS: Genomic DNA amplification by the multiple displacement amplification (MDA) method. Biochem Soc Trans 2009, 37(Pt 2):450-453

17. Lovmar L, Syvanen AC: Multiple displacement amplification to create a long-lasting source of DNA for genetic studies. Hum Mutat 2006, 27(7):603-614.

18. Anjum GM, Du W, Klein R, Amara U, Huber-Lang M, Schneider EM, Wiegand P: Pyrosequencing-based strategy for a successful SNP detection in two hypervariable regions: $\mathrm{HV}-\mathrm{I} / \mathrm{HV}-\mathrm{II}$ of the human mitochondrial displacement loop. Electrophoresis 2010, 31(2):309-314.

19. Xing J, Watkins WS, Zhang Y, Witherspoon DJ, Jorde LB: High fidelity of whole-genome amplified DNA on high-density single nucleotide polymorphism arrays. Genomics 2008, 92(6):452-456.

20. Croft DT Jr, Jordan RM, Patney HL, Shriver CD, Vernalis MN, Orchard TJ, Ellsworth DL: Performance of whole-genome amplified DNA isolated from serum and plasma on high-density single nucleotide polymorphism arrays. J Mol Diagn 2008, 10(3):249-257.

21. Barker DL, Hansen MS, Faruqi AF, Giannola D, Irsula OR, Lasken RS, Latterich M, Makarov V, Oliphant A, Pinter JH, et al: Two methods of whole-genome amplification enable accurate genotyping across a 2320-SNP linkage panel. Genome Res 2004, 14(5):901-907. 
22. Spits C, Le Caignec C, De Rycke M, Van Haute L, Van Steirteghem A, Liebaers I, Sermon K: Whole-genome multiple displacement amplification from single cells. Nat Protoc 2006, 1(4):1965-1970.

23. Coskun $\mathrm{S}$, Alsmadi $\mathrm{O}$ : Whole genome amplification from a single cell: a new era for preimplantation genetic diagnosis. Prenat Diagn 2007, 27(4):297-302.

24. Jiang Z, Zhang $X$, Deka $R$, Jin L: Genome amplification of single sperm using multiple displacement amplification. Nucleic Acids Res 2005, 33(10):e91.

25. Handyside AH, Robinson MD, Simpson RJ, Omar MB, Shaw MA, Grudzinskas $J G$, Rutherford A: Isothermal whole genome amplification from single and small numbers of cells: a new era for preimplantation genetic diagnosis of inherited disease. Mol Hum Reprod 2004, 10(10):767-772

26. Hellani A, Coskun S, Tbakhi A, Al-Hassan S: Clinical application of multiple displacement amplification in preimplantation genetic diagnosis. Reprod Biomed Online 2005, 10(3):376-380.

27. Giardina E, Pietrangeli I, Martone C, Zampatti S, Marsala P, Gabriele L, Ricci O, Solla G, Asili P, Arcudi G, et al: Whole genome amplification and real-time PCR in forensic casework. BMC Genomics 2009, 10:159.

28. Schneider PM, Balogh K, Naveran N, Bogus M, Bender K, Lareu M, Carracedo $\mathrm{A}$ : Whole genome amplification - the solution for a common problem in forensic casework? Int Congr Ser 2004, 1261:24-26.

29. Ballantyne KN, van Oorschot RA, Mitchell RJ: Comparison of two whole genome amplification methods for STR genotyping of LCN and degraded DNA samples. Forensic Sci Int 2007, 166(1):35-41.

30. Le Caignec C, Spits C, Sermon K, De Rycke M, Thienpont B, Debrock S, Staessen C, Moreau Y, Fryns JP, Van Steirteghem A, et al: Single-cell chromosomal imbalances detection by array CGH. Nucleic Acids Res 2006, 34(9):e68.

31. Bergen AW, Qi Y, Haque KA, Welch RA, Chanock SJ: Effects of DNA mass on multiple displacement whole genome amplification and genotyping performance. BMC Biotechnol 2005, 5:24.

32. Liang CW, Lee YS, Marino-Enriquez A, Tsui K, Huang SH: The utility and limitation of single nucleotide polymorphism analysis on whole genome amplified mesenchymal tumour DNA in formalin fixed tumour samples. Pathology 2012, 44(1):33-41.

33. Paez JG, Lin M, Beroukhim R, Lee JC, Zhao X, Richter DJ, Gabriel S, Herman $P$, Sasaki $H$, Altshuler $D$, et al: Genome coverage and sequence fidelity of phi29 polymerase-based multiple strand displacement whole genome amplification. Nucleic Acids Res 2004, 32(9):e71

34. Pinard R, de Winter A, Sarkis GJ, Gerstein MB, Tartaro KR, Plant RN, Egholm $M$, Rothberg JM, Leamon $J H$ : Assessment of whole genome amplificationinduced bias through high-throughput, massively parallel whole genome sequencing. BMC Genomics 2006, 7:216.

35. Arriola E, Lambros MB, Jones C, Dexter T, Mackay A, Tan DS, Tamber N Fenwick K, Ashworth A, Dowsett M, et al: Evaluation of Phi29-based whole-genome amplification for microarray-based comparative genomic hybridisation. Lab Invest 2007, 87(1):75-83.

36. Kallioniemi A, Kallioniemi OP, Sudar D, Rutovitz D, Gray JW, Waldman F, Pinkel D: Comparative genomic hybridization for molecular cytogenetic analysis of solid tumors. Science 1992, 258(5083):818-821.

37. Corey M, McLaughlin FJ, Williams M, Levison $\mathrm{H}$ : A comparison of survival, growth, and pulmonary function in patients with cystic fibrosis in Boston and Toronto. J Clin Epidemiol 1988, 41(6):583-591.

38. Lander ES: Initial impact of the sequencing of the human genome. Nature 2011, 470(7333):187-197.

39. Hamburg MA, Collins FS: The path to personalized medicine. N Engl J Med 2010, 363(4):301-304.

40. Cooper DN, Chen JM, Ball EV, Howells K, Mort M, Phillips AD, Chuzhanova N, Krawczak M, Kehrer-Sawatzki H, Stenson PD: Genes, mutations, and human inherited disease at the dawn of the age of personalized genomics. Hum Mutat 2010, 31(6):631-655.

41. Chen B, Gagnon M, Shahangian S, Anderson NL, Howerton DA, Boone JD: Good laboratory practices for molecular genetic testing for heritable diseases and conditions. MMWR Recomm Rep 2009, 58(RR-6):1-37. quiz CE-31-34.

42. Secretary's Advisory Committee on Genetics, Health, and Society: U.S. System of Oversight of Genetic Testing: A Response to the Charge of the Secretary of Health and Human Services. Bethesda, MD: Department of Health and Human Services. 2008:276. http://oba.od.nih.gov/oba/sacghs/reports/ sacghs_oversight_report.pdf.
43. Amos J, Grody W: Development and integration of molecular genetic tests into clinical practice: the US experience. Expert Rev Mol Diagn 2004 4(4):465-477.

44. Moskowitz SM, Chmiel JF, Sternen DL, Cheng E, Gibson RL, Marshall SG, Cutting GR: Clinical practice and genetic counseling for cystic fibrosis and CFTR-related disorders. Genet Med 2008, 10(12):851-868.

45. Venkatraman ES, Olshen AB: A faster circular binary segmentation algorithm for the analysis of array CGH data. Bioinformatics 2007, 23(6):657-663.

46. Olshen $A B$, Venkatraman ES, Lucito $R$, Wigler M: Circular binary segmentation for the analysis of array-based DNA copy number data. Biostatistics 2004, 5(4):557-572.

47. Huber PJ: Robust Statistics. New York: John Wiley and Sons press; 1981

48. Cramer H: Mathematical Methods of Statistics. New Jersey: Princeton University Press; 1946.

doi:10.1186/1471-2164-13-217

Cite this article as: Han et al:: Characterization of whole genome amplified (WGA) DNA for use in genotyping assay development. BMC Genomics 2012 13:217.

\section{Submit your next manuscript to BioMed Central and take full advantage of:}

- Convenient online submission

- Thorough peer review

- No space constraints or color figure charges

- Immediate publication on acceptance

- Inclusion in PubMed, CAS, Scopus and Google Scholar

- Research which is freely available for redistribution 